\title{
Pediatric and adult dilated cardiomyopathy represent distinct pathological entities
}

\author{
Meghna D. Patel, ${ }^{1}$ Jayaram Mohan, ${ }^{2}$ Caralin Schneider, ${ }^{2}$ Geetika Bajpai, ${ }^{2}$ Enkhsaikhan Purevjav, ${ }^{3}$ \\ Charles E. Canter, Jeffrey Towbin, ${ }^{3}$ Andrea Bredemeyer, ${ }^{2}$ and Kory J. Lavine ${ }^{2,4,5}$ \\ 'Department of Pediatrics, ${ }^{2}$ Center for Cardiovascular Research, Division of Cardiology, Department of Medicine, \\ Washington University School of Medicine, St. Louis, Missouri, USA. ${ }^{3}$ Department of Pediatrics, Division of Cardiology, \\ University of Tennessee Health Science Center, Memphis, Tennessee, USA. ${ }^{4}$ Department of Developmental Biology, and \\ ${ }^{5}$ Department of Immunology and Pathology, Washington University School of Medicine, St. Louis, Missouri, USA
}

Pediatric dilated cardiomyopathy (DCM) is the most common indication for heart transplantation in children. Despite similar genetic etiologies, medications routinely used in adult heart failure patients do not improve outcomes in the pediatric population. The mechanistic basis for these observations is unknown. We hypothesized that pediatric and adult DCM comprise distinct pathological entities, in that children do not undergo adverse remodeling, the target of adult heart failure therapies. To test this hypothesis, we examined LV specimens obtained from pediatric and adult donor controls and DCM patients. Consistent with the established pathophysiology of adult heart failure, adults with DCM displayed marked cardiomyocyte hypertrophy and myocardial fibrosis compared with donor controls. In contrast, pediatric DCM specimens demonstrated minimal cardiomyocyte hypertrophy and myocardial fibrosis compared with both age-matched controls and adults with DCM. Strikingly, RNA sequencing uncovered divergent gene expression profiles in pediatric and adult patients, including enrichment of transcripts associated with adverse remodeling and innate immune activation in adult DCM specimens. Collectively, these findings reveal that pediatric and adult DCM represent distinct pathological entities, provide a mechanistic basis to explain why children fail to respond to adult heart failure therapies, and suggest the need to develop new approaches for pediatric DCM.

Conflict of interest: The authors have declared that no conflict of interest exists.

Submitted: April 3, 2017

Accepted: June 6, 2017

Published: July 20, 2017

Reference information: JCI Insight. 2017;2(14):e94382 https://doi.org/10.1172/jici. insight.94382.

\section{Introduction}

Dilated cardiomyopathy (DCM) is an important cause of childhood mortality and is the most common indication for heart transplantation in the pediatric population (1-3). Despite efforts to improve patient care, pediatric DCM remains a challenging disease with an estimated 50\% 5-year transplant-free survival (1). Within both the pediatric and adult cohorts, DCM is often associated with mutations in sarcomeric and cytoskeletal genes (4). While the genetic basis for pediatric DCM is increasingly recognized (4), few therapies have been developed for children suffering from this devastating disease.

Landmark clinical trials have led to the establishment of medical therapies for adults with dilated and ischemic cardiomyopathy (5). Collectively, these therapies target a process termed adverse remodeling, a common pathway by which the adult heart responds to injury. Pathologically, adverse remodeling is defined by cardiomyocyte hypertrophy, myocardial fibrosis, inflammation, and capillary loss (6). While it is reasonable to postulate that adult heart failure therapies may be efficacious in children, clinical trials have not supported this idea. Despite marked reductions in mortality associated with $\beta$-blocker therapy in adult studies, the Pediatric Carvedilol Trial failed to demonstrate improvements in clinical outcomes in children with symptomatic heart failure $(7,8)$. In fact, since the implementation of digoxin and diuretic therapies decades ago, there has been little improvement in outcomes for children with DCM (9). These observations question the effectiveness of antiremodeling therapies in children with heart failure and support the idea that, despite a common genetic etiology (4), pediatric and adult DCM may represent distinct pathologic entities.

One potential explanation for why antiremodeling therapeutics are not effective in the pediatric population is that adverse remodeling does not govern disease progression in children. Consistent with this 
Table 1. Pediatric and adult DCM demographic data

\begin{tabular}{|c|c|c|c|}
\hline & Pediatric DCM & Adult DCM & $P$ value \\
\hline$n=$ & 31 & 34 & \\
\hline Age in yrs & 6.5 & 51.0 & $<0.01$ \\
\hline Male & 21/31 (67.7) & $28 / 34(82.4)$ & 0.17 \\
\hline \multicolumn{4}{|l|}{ Diagnosis } \\
\hline Idiopathic & $29 / 31(93.5)$ & $30 / 34(88.2)$ & 0.47 \\
\hline Familial & $2 / 31(6.5)$ & 4/34 (11.8) & 0.46 \\
\hline \multicolumn{4}{|l|}{ Tissue source } \\
\hline LVAD core & 6/31 (19.4) & 29/34 (85.3) & $<0.01$ \\
\hline Heart explant & $25 / 31$ (80.6) & $5 / 34(14.7)$ & $<0.01$ \\
\hline Age at diagnosis (yrs) & 7.3 & 46.0 & $<0.01$ \\
\hline Disease duration (yrs) & 2.4 & 5.7 & 0.02 \\
\hline Hypertension & $1 / 31(3.2)$ & $15 / 34(44.1)$ & $<0.01$ \\
\hline Diabetes & $0 / 31(0)$ & $13 / 34(38.2)$ & $<0.01$ \\
\hline Chronic kidney disease & $0 / 31(0)$ & 6/34 (17.6) & $<0.01$ \\
\hline \multicolumn{4}{|l|}{ Medications } \\
\hline$\beta$-Blockers & $4 / 8(50.0)$ & 31/34 (91.1) & $<0.01$ \\
\hline ACEi/ARB & $4 / 8(50.0)$ & $29 / 34(85.3)$ & 0.03 \\
\hline MRAs & $2 / 8(25.0)$ & $21 / 34(61.2)$ & 0.07 \\
\hline Diuretics & $3 / 8(37.5)$ & $33 / 34(97.1)$ & $<0.01$ \\
\hline Digoxin & $3 / 8(37.5)$ & $13 / 34(38.2)$ & 0.97 \\
\hline Hydralazine & $0 / 8(0)$ & $7 / 34(20.6)$ & 0.16 \\
\hline Nitrates & $0 / 8(0)$ & $8 / 34(23.5)$ & 0.13 \\
\hline \multicolumn{4}{|l|}{ Echocardiography } \\
\hline EF (\%) [Z score] & $22.5[-7.6]$ & 17.2 & 0.01 \\
\hline FS (\%) [Z score] & $11.5[-14.2]$ & 8.9 & 0.12 \\
\hline LVDD (mm) [Z score] & $5.4[9.0]$ & 7.0 & N/A \\
\hline LVSD (mm) [Z score] & $4.8[13.7]$ & 6.4 & $\mathrm{~N} / \mathrm{A}$ \\
\hline
\end{tabular}

Percentages displayed in parenthesis. $Z$ scores displayed in brackets for pediatric echocardiographic data. EF, ejection fraction; FS, fractional shortening; LVDD, left ventricular diastolic dimension (m-mode); LVSD, left ventricular systolic dimension ( $m$-mode); CKD, defined as stage 3 or greater chronic kidney disease; ACEi, angiotensin converting enzyme inhibitor; ARB, angiotensin II receptor blocker; MRA, mineralocorticoid receptor antagonist. Pediatric echocardiography data represents a limited data set derived from 10 specimens. All other data is derived from the complete sample set. hypothesis, some hallmarks of adverse remodeling including $\beta$-adrenergic receptor downregulation, and upregulation of fetal gene expression appears to occur to a lesser extent in pediatric compared with adult heart failure specimens (10). Intriguingly, no studies have rigorously determined whether adverse remodeling occurs in children or deciphered at what age adverse remodeling occurs in adults. Moreover, there is a paucity of information delineating the molecular differences that define pediatric and adult heart failure.

To test the hypothesis that adverse remodeling does not drive disease progression in pediatric DCM, we obtained cardiac tissue specimens from the left ventricle (LV) of pediatric and adult patients with familial and idiopathic DCM. Using a combination of quantitative histology, immunostaining, electron microscopy, and RNA sequencing, we show that pediatric DCM patients do not display pathologic evidence of adverse remodeling compared with age-matched donor control specimens. In contrast, adult patients uniformly displayed pathological evidence of adverse remodeling. Consistent with pathological evidence of adverse remodeling in adult DCM specimens, RNA sequencing revealed that genes associated with sarcomere remodeling, inflammation, and fatty acid metabolism were enriched in adult compared with pediatric DCM specimens. Collectively, these findings indicate that adverse remodeling does not occur in children with DCM and suggest that pediatric and adult DCM may represent distinct pathological entities.

\section{Results}

Study design. To test the hypothesis that adverse remodeling does not occur in children with DCM, we obtained LV myocardial specimens from the apex and lateral wall of nonfailing pediatric donor controls $(n=11)$, pediatric DCM patients $(n=$ 31 ), adult nonfailing donor controls $(n=14)$, and adult DCM patients $(n=34)$ (Table 1 and Supplemental Figure 1 ; supplemental material available online with this article; https://doi. org/10.1172/jci.insight.94382DS1). To our knowledge, this represents the largest collection of pediatric and adult DCM

tissue specimens studied to date. Control tissue was obtained from unused donors with normal cardiac function by echocardiography (Table 2). DCM specimens were obtained from children and adults with advancedstage idiopathic or familial DCM at the time of cardiac transplantation or implantation of a LV assist device. Diseased tissue specimens were further subclassified into age groups of $0-1$ years (yrs) $(n=9), 1-10$ yrs $(n=$ $12), 11-18$ yrs $(n=10), 19-50$ yrs $(n=15)$, and $51-75$ yrs $(n=19)$. To determine whether adverse remodeling occurs in pediatric DCM, we measured cardiomyocyte and sarcomere size, myocardial fibrosis, and capillary density in pediatric and adult donor control and DCM specimens. In addition, we performed RNA sequencing on pediatric and adult DCM specimens to examine gene expression signatures of adverse remodeling and define pathways that differentiate pediatric from adult DCM.

Demographics. Clinical data obtained from pediatric and adult DCM patients demonstrated similar distributions of male and female subjects and comparable numbers of subjects with idiopathic and familial DCM (Table 1). Significant differences included the number of left ventricular assist device (LVAD) core and heart explant specimens. The pediatric DCM group had a greater number of heart explant specimens, while the adult DCM group had a greater number of LVAD core specimens. In addition, disease duration was longer in adult DCM subjects, and the frequency of patients with cardiovascular comorbidities - including hypertension, chronic kidney disease, and diabetes - was more prevalent in adult patients with DCM. As expected, adult DCM patients were more likely to have received antiremodeling therapies. Echocardiographic data confirmed depressed ejec- 
A
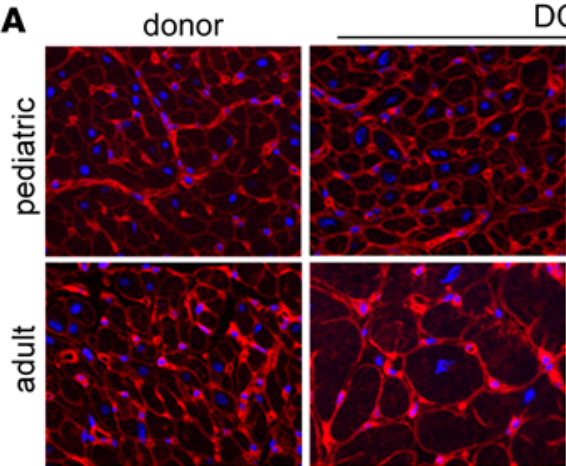

DCM

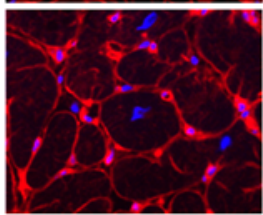

B
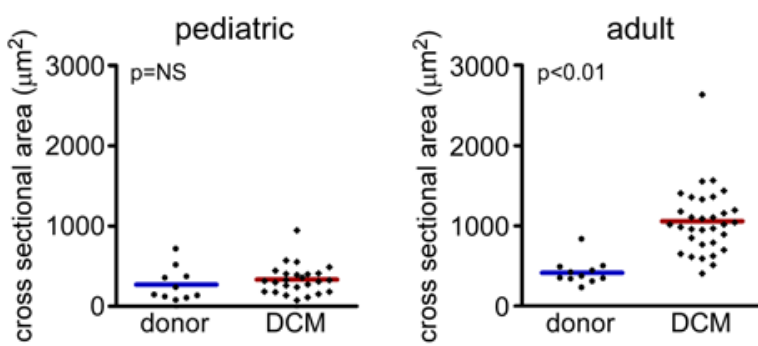

C

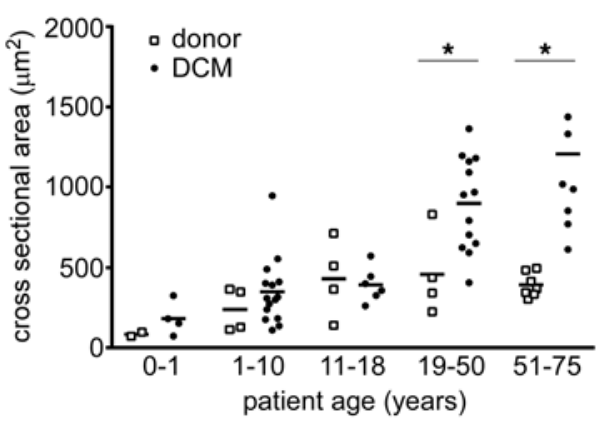

D
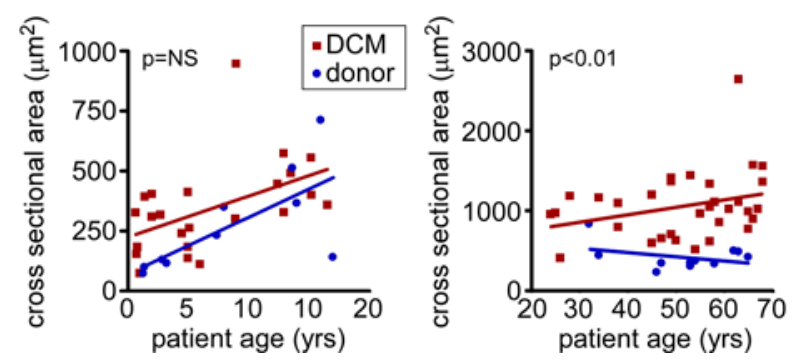

Figure 1. Absence of cardiomyocyte hypertrophy in pediatric DCM. (A) Wheat germ agglutinin (WGA) staining (red) demonstrating that pediatric donor controls and pediatric DCM specimens display similar cardiomyocyte cross-sectional area. In contrast, adult DCM patients display increased cardiomyocyte cross-sectional area compared with donor controls. Blue, DAPI (nuclei). Magnification 400x. (B) Quantification of cardiomyocyte cross-sectional area. (C) Cardiomyocyte cross-sectional area stratified by age, revealing that cardiomyocyte cross-sectional area does not differ from donor controls across pediatric groups. ${ }^{*} P<0.05$ (Mann Whitney $U$ test). (D) Scatter plots showing that cardiomyocyte cross-sectional area does not differ as a function of age in pediatric DCM patients. In contrast, adult DCM patients have increased cardiomyocyte cross-sectional area at all ages examined.

tion fraction and fractional shortening, as well as increased LV systolic and diastolic chamber dimensions in both the adult and pediatric DCM groups. LV ejection fraction was slightly lower in the adult DCM cohort. Examination of available clinical data obtained from pediatric and adult donor controls demonstrated similar sex distribution and ejection fraction. There were a higher proportion of adult donor controls who had stroke listed as the cause of brain death (Table 2).

Cardiomyocyte hypertrophy. To examine the extent of cardiomyocyte hypertrophy, we stained pediatric and adult donor control and DCM specimens with rhodamine conjugated wheat germ agglutinin (WGA) and measured cardiomyocyte cross-sectional area (Figure 1A). Measurements were made perpendicular to the long axis of each cardiomyocyte. Compared with donor control, pediatric DCM specimens did not demonstrate evidence of increased cardiomyocyte cross-sectional area $\left(271.8 \pm 210\right.$ vs. $\left.333.5 \pm 184 \mu \mathrm{m}^{2}, P=0.24\right)$. Consistent with the presence of cardiomyocyte hypertrophy in adult DCM, WGA staining revealed a marked increase in cardiomyocyte cross-sectional area in adult DCM samples compared with donor controls (416.5 \pm 159 vs. $1,058 \pm 420 \mu \mathrm{m}^{2}, P<0.001$ ) (Figure 1B). Stratification of donor controls and DCM specimens by age group further demonstrated that increased cardiomyocyte cross-sectional area was only evident in adult age groups (Figure 1C). Stratification of pediatric samples by age group $(0-1,1-10,11-18$ yrs ) demonstrated no differences in cardiomyocyte cross-sectional area between donor control and pediatric DCM samples. In contrast, adult DCM samples displayed increased cardiomyocyte cross-sectional area in 19-50 and 51-65 yrs age groups. Linear regression analysis further confirmed that only adult DCM samples displayed increased cardiomyocyte cross-sectional area throughout the spectrum of ages examined (Figure 1D).

To provide additional evidence that cardiomyocyte hypertrophy preferentially occurs in adult and not pediatric DCM, we examined cardiomyocyte ultrastructure using transmission electron microscopy (Figure 2A). Previous studies have established that pathologic cardiomyocyte hypertrophy is associated with increased sarcomere thickness $(11,12)$. Compared with donor controls, pediatric DCM specimens did not demonstrate any changes in sarcomere thickness $(0.54 \pm 0.04$ vs. $0.62 \pm 0.14 \mu \mathrm{m}, P=0.12)$. In contrast, adult DCM specimens displayed increased sarcomere thickness $(0.76 \pm 0.12$ vs. $1.25 \pm 0.27 \mu \mathrm{m}$, $P<0.01$ ) compared with donor controls (Figure $2 \mathrm{~B}$ ).

Myocardial fibrosis. To determine whether pediatric and adult DCM specimens display evidence of myocardial fibrosis compared with age-matched controls, we performed Picrosirius red staining, a specific marker of collagen deposition (13). As anticipated, pediatric and adult donor control specimens displayed Picrosirius red staining surrounding larger arteries and small blood vessels (perivascular space) and minimal staining around individual cardiomyocytes (interstitial space). Pediatric DCM specimens displayed similar degrees of perivascular fibrosis and minimal interstitial fibrosis. In contrast, adult DCM specimens 
Table 2. Donor control demographic data

\begin{tabular}{lccc}
\hline & Pediatric donor & Adult donor & P value \\
$\boldsymbol{n}=$ & 11 & 14 & \\
Age in yrs & 11.3 & 50.0 & $<0.01$ \\
Male & $7 / 11(63.6)$ & $9 / 14(64.3)$ & 0.97 \\
EF (\%) & 54.0 & 61.7 & 0.71 \\
Cause of death & & & \\
Head trauma & $4 / 11(36.4)$ & $4 / 14(28.6)$ & 0.68 \\
$\quad$ Stroke & $0 / 11(0)$ & $6 / 14(42.9)$ & 0.01 \\
$\quad$ Unknown & $7 / 11(63.6)$ & $4 / 14(28.6)$ & 0.09
\end{tabular}

Percentages displayed in parenthesis. EF, ejection fraction. demonstrated patchy areas of dense interstitial fibrosis and accentuated perivascular fibrosis compared with donor controls (Figure 3A).

Given the presence of two distinct patterns of myocardial fibrosis (interstitial and perivascular), we chose to employ two methods to quantify myocardial fibrosis: i) total Picrosirius red staining, and ii) a semiquantitative scoring system to individually quantify the extent of perivascular and interstitial fibrosis (Supplemental Figure 2). To minimize potential bias, specimens were scored by two independent investigators blinded to sample identity. Quantification of the percentage of Picrosirius red staining per $20 \times$ field revealed no evidence of myocardial fibrosis in pediatric DCM specimens compared with pediatric donor controls $(1.7 \pm 0.7$ vs. $2.0 \pm 0.3, P=0.46)$. In contrast, adult DCM specimens displayed evidence of marked myocardial fibrosis compared with adult donor controls ( $1.8 \pm 0.2$ vs. $12.3 \pm$

$1.1, P<0.01)$ (Figure 3B). Quantification of fibrosis scores revealed no evidence of interstitial $(0.10$ \pm 0.3 vs. $0.41 \pm 0.8, P=0.08)$ or perivascular fibrosis $(1.1 \pm 0.7$ vs. $0.52 \pm 0.7, P=0.05)$ in pediatric DCM specimens compared with pediatric donor controls. In contrast, adult DCM specimens displayed evidence of increased interstitial $(0.2 \pm 0.6$ vs. $1.8 \pm 0.8, P<0.01)$ and perivascular $(1.1 \pm 0.8$ vs. $1.6 \pm$ $0.7, P=0.03$ ) fibrosis compared with adult donor controls (Figure $3 \mathrm{C}$ ). Stratification of pediatric and adult DCM samples by patient age using linear regression and subgroup analyses demonstrated minimal total, interstitial, or perivascular fibrosis across the spectrum of pediatric age groups $(0-1,1-10$, and 10-18 yrs). In contrast, prominent total, interstitial, and perivascular fibrosis was found in each adult subgroup (19-50 and 51-75 yrs) consistent with prior reports (14) (Figure 3, D-F).

Coronary microvasculature. While conflicting data exist regarding whether coronary microvascular density is reduced in adult patients with chronic DCM (15-17), it is well appreciated that reductions in myocardial perfusion and flow reserve are strong predictors of poor clinical outcomes (18-22). To date, few studies have examined whether coronary microvascular density is altered in pediatric DCM. To clarify whether coronary microvascular density is affected in pediatric DCM, we performed CD34 immunostaining on pediatric and adult donor control and DCM specimens. Microvascular density was quantified by measuring the percentage of the myocardium that displayed CD34 staining, a method we have previously demonstrated to tightly correlate with the number of capillaries present per high-power field (23).

Compared with pediatric donor controls, coronary microvascular density was increased in pediatric DCM $(6.1 \% \pm 0.9 \%$ vs. $10.9 \% \pm$ $5.5 \%, P<0.001)$. In contrast, adult donor controls and adult DCM specimens displayed similar microvascular density $(6.7 \% \pm 1.7 \%$ vs. $6.5 \% \pm 2.0 \%, P=0.72$ ) (Figure 4, $\mathrm{A}$ and $\mathrm{B}$ ). When CD34 staining was further stratified by age groups using either linear regression or subgroup analyses, we found that coronary microvascular density was increased across the spectrum of pediatric ages. In contrast, coronary microvascular density was indistinguishable from adult donor controls across each adult DCM age group (Figure 4, C and D).

Figure 2. Sarcomere structure in pediatric and adult DCM. (A) Electron microscopy examining sarcomere structure in donor control, pediatric, and adult DCM specimens. Compared with donor controls, pediatric DCM patients display no change in sarcomere thickness. In contrast, adult DCM patients demonstrate increased sarcomere thickness compared with donor controls. (B) Quantification of sarcomere thickness. Each data point represents an average of $>20$ sarcomeres measured from individual patient samples. $P$ values found with Mann Whitney $U$ test.

\section{A}
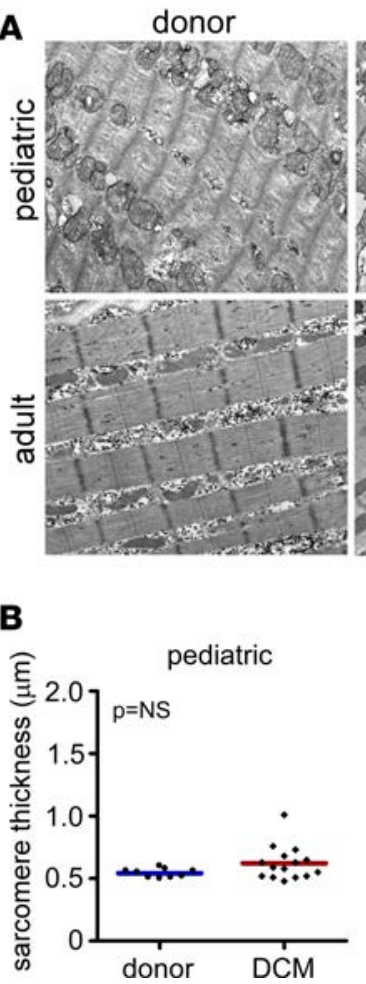
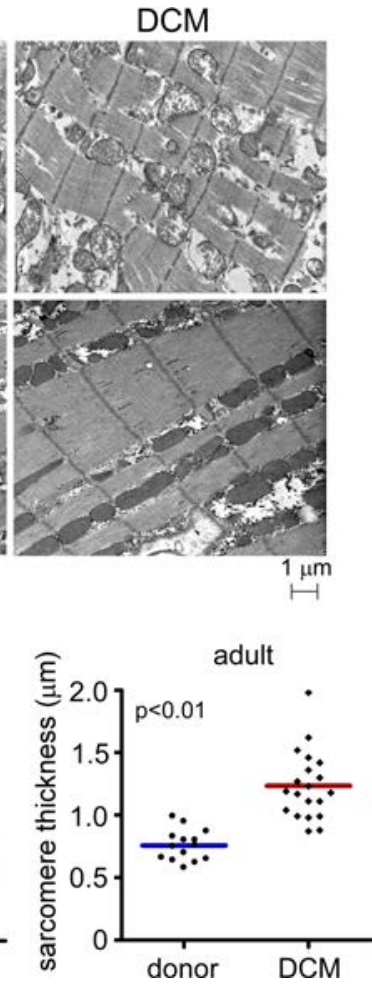
A
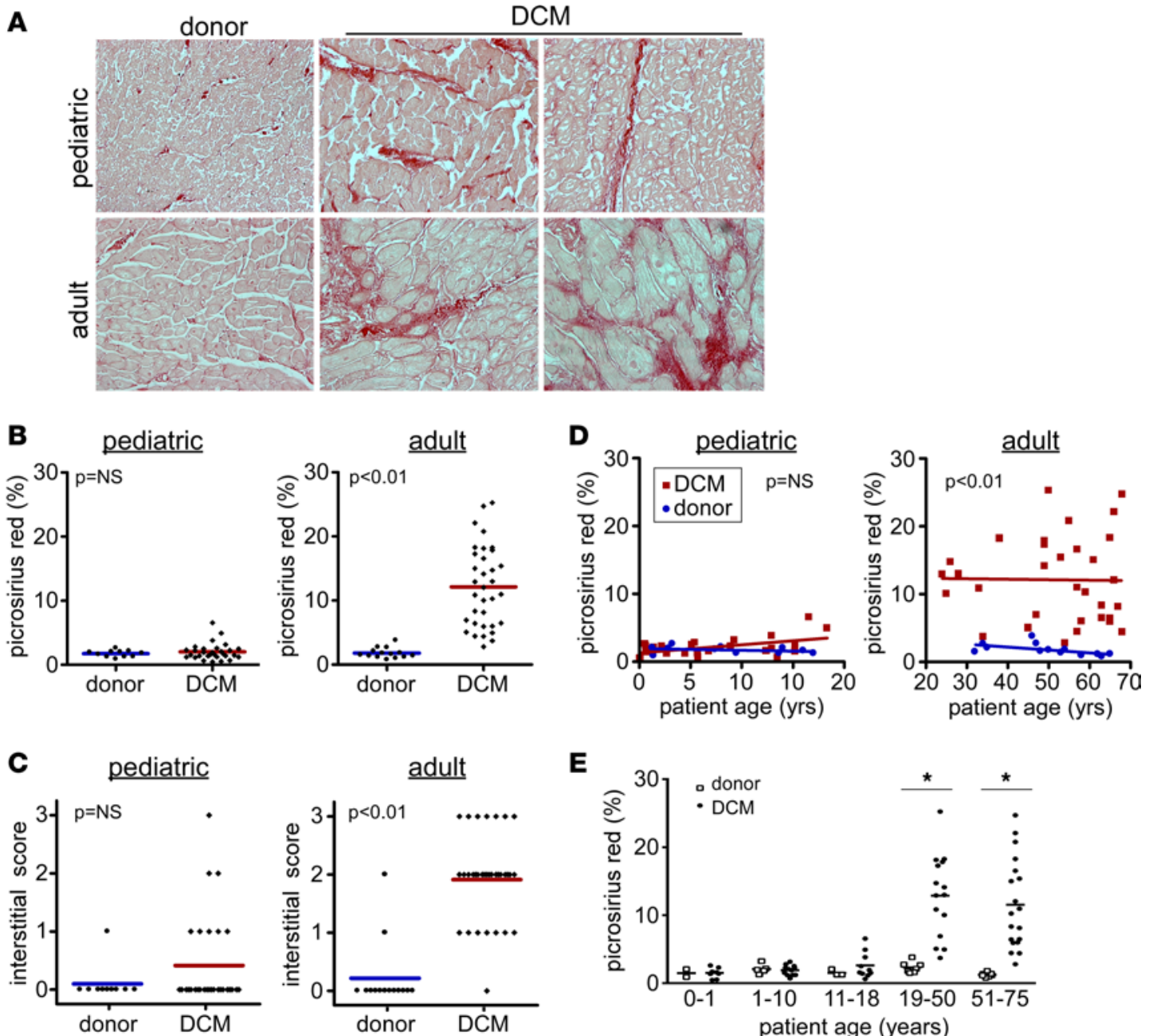

\section{$\mathbf{E}$}
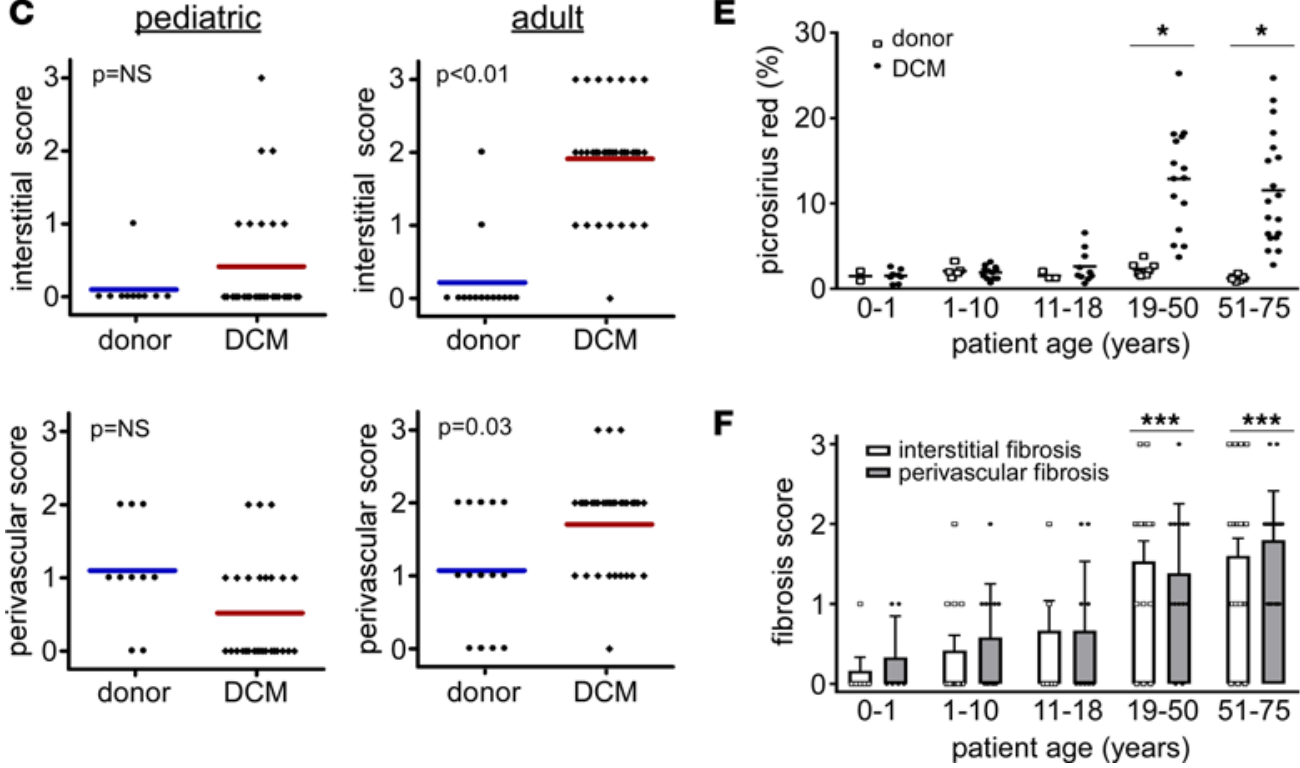

Figure 3. Absence of myocardial fibrosis in pediatric DCM. (A) Picrosirius red staining (red) demonstrating that, compared with donor controls, pediatric DCM specimens display minimal interstitial or perivascular fibrosis. In contrast, adult DCM patients display increased interstitial and perivascular fibrosis compared with donor controls. Magnification 200x. (B) Quantification of percent fibrosis as assessed by total Picrosirius red staining per 20x field in pediatric and adult control and DCM specimens. (C) Quantification of interstitial and perivascular fibrosis scores. (D) Scatter plots showing that the percent of Picrosirius staining in pediatric DCM specimens does not differ from donor pediatric controls across the spectrum of child age. In contrast, adult DCM patients have increased Picrosirius red staining at all ages examined. (E and $\mathbf{F})$ Total Picrosirius red staining $(\mathbf{E})$ and fibrosis scores $(\mathbf{F})$ stratified by age, revealing that interstitial and perivascular fibrosis scores are only increased in adult groups. ${ }^{*} P<0.05$ ( $\chi^{2}$ test), ${ }^{* * *} P<0.05$ (compared to pediatric groups).

Adverse remodeling, disease duration, and comorbidities. As the duration of disease significantly differs between pediatric and adult DCM, with adult patients having longer periods of disease (24-26) (Table 1), we viewed heart failure duration as a confounding variable that may potentially explain why children with DCM display less adverse remodeling compared with adult DCM patients. To account for disease duration (defined as the period of time between clinical diagnosis of heart failure and myocardial specimen collection), we divided our adult DCM cohort into three groups: disease duration less than 1 year, disease duration $1-5$ years, and disease duration greater than 5 years. 
A

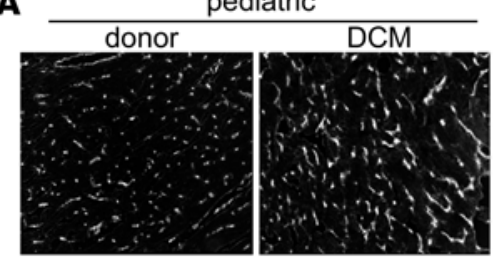

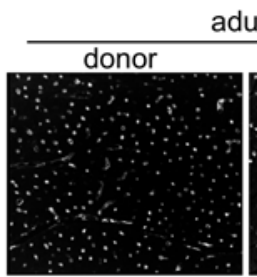

adult

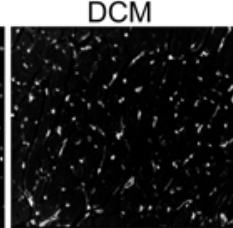

adult

B

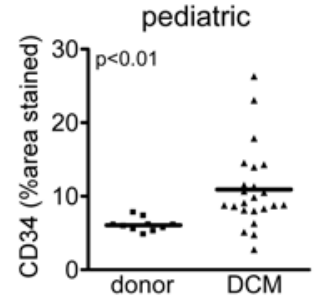

C

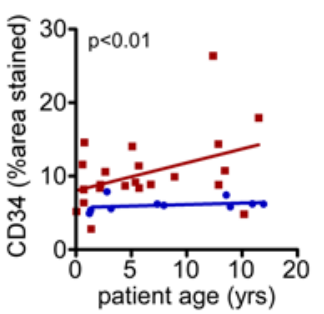

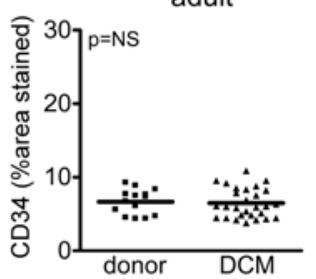

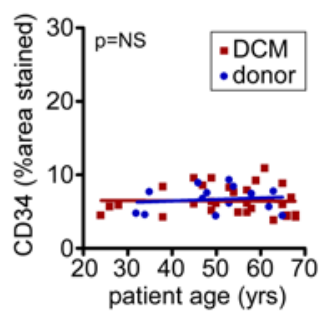

a donor

- DCM

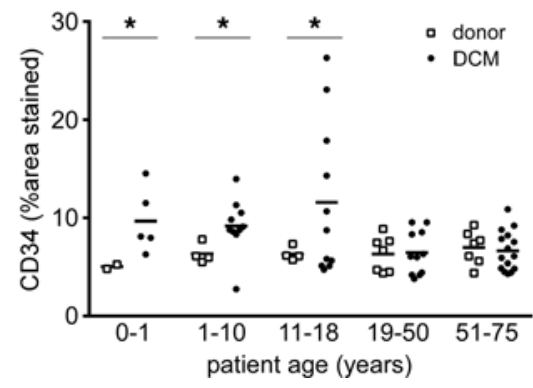

Figure 4. Microvascular alterations in pediatric and adult DCM. (A) CD34 immunostaining demonstrating that, compared with donor controls, pediatric DCM specimens display increased microvascular density. In contrast, adult DCM patients have indistinguishable microvascular density compared with donor controls. Magnification 200x. (B) Quantification of microvascular density expressed as the percent of staining per $20 \times$ field. (C) Scatter plots showing that pediatric DCM patients display increased capillary density compared with donor controls across the spectrum of child ages. In contrast, capillary density did not differ between adult DCM specimens and donor controls at all ages examined. (D) CD34 staining stratified by age, revealing that microvascular density is increased in all pediatric groups. ${ }^{*} P$ $<0.05$ (Mann Whitney $U$ test).

Compared with donor controls, adult DCM patients who were diagnosed with heart failure for less than 1 year displayed similar increases in cardiomyocyte cross-sectional area compared with those diagnosed with heart failure for 1-5 years and greater than 5 years (Figure 5, A and B). While there was a trend for increased cardiomyocyte cross-sectional area with longer disease durations, these relationships were not statistically significant, suggesting that cardiomyocyte hypertrophy occurs largely independent of disease duration. Similarly, the extent of myocardial fibrosis (Figure 5, C-F) and coronary microvascular density (Figure 5, G and $\mathrm{H}$ ) were not influenced by disease duration. Stratification of pediatric DCM patients by disease duration revealed no relationship between disease duration, cardiomyocyte hypertrophy, and myocardial fibrosis (Figure 5, I and J).

As the frequency of hypertension, chronic kidney disease, and diabetes was significantly increased in adult compared with pediatric DCM patients, we performed independent analyses to control for these confounding variables. Compared with donor controls, patients with DCM displayed increased cardiomyocyte hypertrophy and myocardial fibrosis independent of the presence of hypertension, chronic kidney disease, and diabetes (Supplemental Figure 3). Collectively, these data signify that disease duration and cardiovascular comorbidities, including hypertension, chronic kidney disease, and diabetes, are unlikely to account for the observed differences in pathological adverse remodeling between pediatric and adult DCM specimens.

$R N A$ sequencing. To determine whether pediatric and adult DCM represent similar or distinct pathological entities at the transcriptomic level, we performed RNA sequencing. Of the LV myocardial tissues included in this study, 18 pediatric and 33 adult DCM specimens were of suitable quality for RNA sequencing and subsequent analysis (Table 3). Of note, we chose not to include donor control samples in our RNA sequencing experimental design, as these specimens are obtained from donor hearts that are not suitable for transplantation due to prolonged ischemic times, profound end organ failure, advanced age, hepatitis $\mathrm{C} / \mathrm{HIV}$, and preexisting cardiac disease. With respect to this study, we excluded donors with hepatitis C, HIV, and preexisting cardiac disease. Importantly, brain death itself has been demonstrated to increase inflammation within the heart $(27,28)$. Thus, donor specimens obtained from brain-dead individuals are unlikely to represent normal cardiac tissue. While these tissues do not appear to demonstrate chronic pathological changes, they likely have significant derangements in gene expression and would potentially compromise our RNA sequencing analysis. For this reason, we have excluded such controls and recognize age as a potential confounding variable.

In support of the hypothesis that pediatric and adult DCM represent distinct pathological entities, principal component analysis demonstrated clear distinctions between pediatric and adult groups (Figure 6A). Similarly, differential gene expression analysis identified numerous transcripts that were selectively regulated in pediatric and adult DCM specimens across the entire spectrum of transcript abundance (Figure 6B). Among the differentially regulated transcripts, 387 mRNAs were upregulated in pediat- 
Table 3. RNA sequencing analysis

\begin{tabular}{lccc}
\hline & Pediatric DCM & adult DCM & P value \\
Total input reads & $38,183,302.6$ & $38,260,819.6$ & 0.96 \\
Uniquely mapped reads & $32,244,707.4$ & $32,504,594.3$ & 0.84 \\
Uniquely mapped reads (\%) & 84.5 & 84.9 & 0.63 \\
Mismatch rate per base (\%) & 0.16 & 0.12 & $<0.01$ \\
Reads mapped to multiple loci & $4,832,155.6$ & $4,961,119.8$ & 0.76 \\
Reads mapped to multiple loci (\%) & 12.5 & 12.9 & 0.64 \\
Unmapped reads (\%) & 2.1 & 1.4 & 0.07
\end{tabular}

ric DCM and 223 mRNA were upregulated in adult DCM by greater than or equal to 2-fold. Likewise, 915 mRNAs were upregulated in pediatric DCM and 1,018 mRNAs were upregulated in adult DCM by greater than or equal to 1.5 -fold (Figure $6 \mathrm{C}$ ).

Consistent with the observation that adverse remodeling selectively occurs in adult DCM, genes previously associated with pathological cardiomyocyte hypertrophy in humans and animal models (Nppa, Myh7, Mybpc3, Actc1, Tpm1, Des, Lmna, My13, Mylk3, Pln, Gdf15, and Mef2) (29-32) were upregulated in adult compared with pediatric DCM. Myh6, a gene that is known to decrease with pathological cardiomyocyte hypertrophy, was downregulated in adult compared with pediatric DCM (Figure 6D). In addition to differential regulation of genes associated with adverse remodeling, Gene Ontology (GO) pathway analysis revealed selective regulation of several other interesting pathways (Table 4). Pathways upregulated in adult DCM included those associated with oxidative reduction, fatty acid metabolism, response to endogenous stimuli and organic substances, and inflammatory/wounding responses. While differences in substrate utilization and $\beta$-adrenergic signaling have been previously described in pediatric and adult heart failure $(10,33,34)$, distinctions in inflammation have yet to be explored. Among the genes associated with inflammation, numerous proinflammatory chemokines, cytokines, danger signals, key signal transduction components, and transcription factors were selectively upregulated in adult DCM (Figure 6E and Supplemental Table 1). Pathways upregulated in pediatric DCM included those previously associated with cell adhesion, ion and transmembrane transport, and visual perception. Among the genes associated with visual perception, many - to our knowledge — represented novel G-protein coupled receptors with similarity to retinal photoreceptors (Supplemental Table 2).

As noted above, we did not include pediatric and adult donor control samples in our analysis. As such, we recognized a priori that age may represent a confounding factor. To evaluate the impact of age on the observed differences between pediatric and adult DCM transcript expression, we performed a hierarchical clustering analysis based on mRNAs differentially expressed between pediatric and adult DCM groups. Importantly, this analysis illustrated that pediatric and adult DCM specimens cluster as two distinct entities rather than as a continuum of age. Within each of the pediatric and adult subgroups, individual specimens are randomly distributed by age (Figure 6F). Collectively, these data support the concept that adverse remodeling selectively occurs in adult DCM and that pediatric and adult DCM represent distinct pathological entities.

\section{Discussion}

This study tested the hypothesis that pediatric and adult DCM represent distinct pathological entities, in that children do not undergo adverse remodeling. Collectively, our data support this hypothesis and provide evidence that distinct pathological mechanisms drive the progression of pediatric and adult DCM. Specifically, we demonstrated that adverse remodeling does not uniformly occur in children with DCM, as myocardial specimens obtained from patients with pediatric DCM display no significant cardiomyocyte hypertrophy, increased sarcomere thickness, or myocardial fibrosis compared with age-matched donor controls. In contrast, myocardial specimens obtained from adults with DCM displayed robust increases in cardiomyocyte size, sarcomere thickness, and myocardial fibrosis compared with age-matched donor controls. Importantly, these findings appear to be irrespective of patient age across pediatric and adult subgroups, and they appear to be independent of disease duration at least within the adult cohort. Consistent with the pathological data, RNA sequencing revealed divergent gene expression profiles between pediatric and adult DCM tissue specimens, with adult specimens displaying marked induction of transcripts associated with adverse remodeling.

To date, this is the first study to have rigorously evaluated whether adverse remodeling occurs in children and to compare myocardial gene expression in a large cohort of pediatric and adult DCM patients. Our results have important clinical implications and may explain several key observations that differentiate pediatric from adult DCM. The establishment of large clinical registries such as the Pediatric Cardiomyopathy Registry (PCMR) and execution of randomized clinical trials has made it possible to define the clinical course and outcomes for pediatric $\operatorname{DCM}(1,7,35,36)$. Children with DCM display remarkable variability in overall outcomes, ranging from complete recovery of cardiac function (referred to as cardiac recovery) 
A

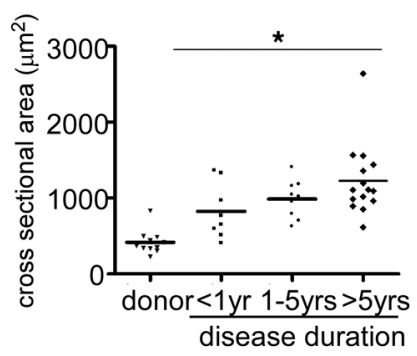

C

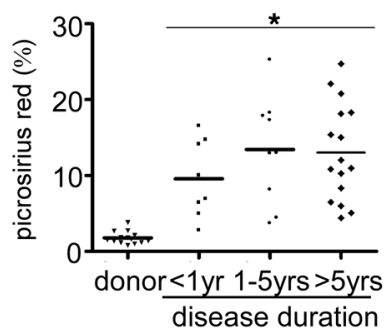

$\mathbf{E}$

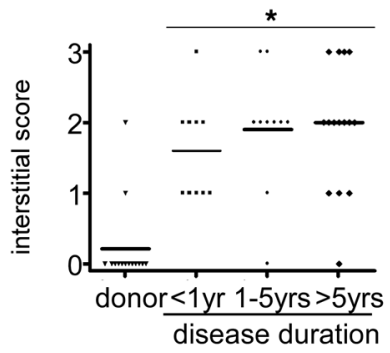

G

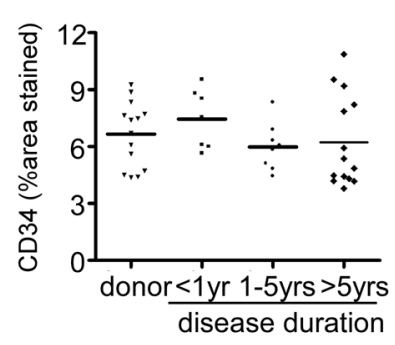

I

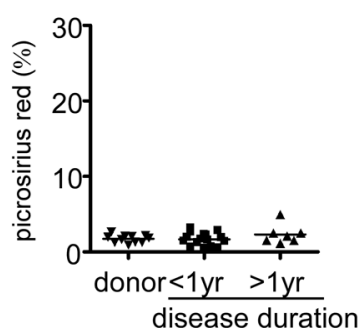

B

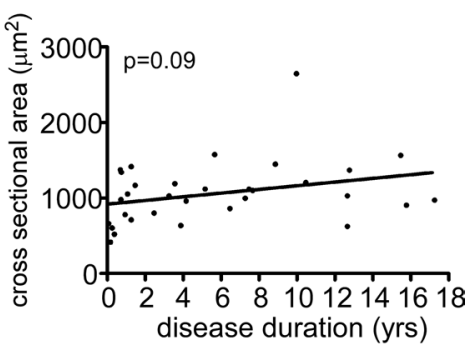

D

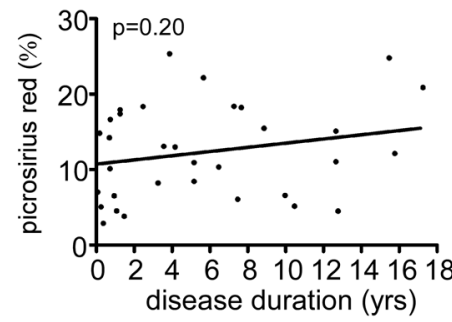

$\mathbf{F}$

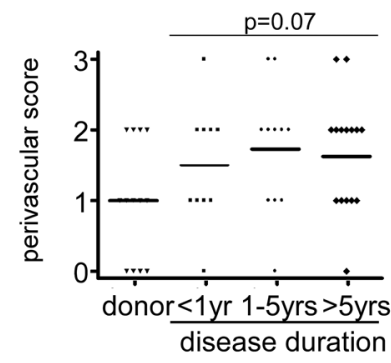

H

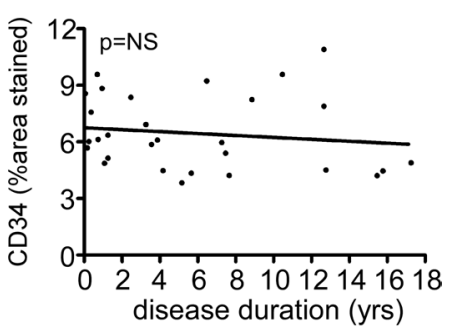

J

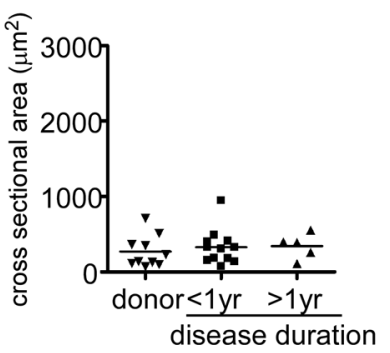

Figure 5. Disease duration does not influence pathological evidence of adverse remodeling in adult and pediatric DCM. (A) Quantification of cardiomyocyte cross-sectional area stratified by disease duration reveals that patients diagnosed with heart failure $<1$ year, $1-5$ years, or $<5$ years prior to tissue procurement displayed marked cardiomyocyte hypertrophy compared with donor controls. No significant differences were evident between disease duration groups. (B) Linear regression analysis describing the relationship between cardiomyocyte cross-sectional area and disease duration. (C) Quantification of total Picrosirius red staining stratified by disease duration reveals that patients diagnosed with heart failure $<1$ year, 1-5 years, or $<5$ years prior to tissue procurement displayed increased fibrosis compared with donor controls. (D) Linear regression analysis describing the relationship between total Picrosirius red staining and disease duration. (E and F) Quantification of interstitial and perivascular fibrosis stratified by disease duration demonstrates that myocardial fibrosis occurs independent of disease duration. No significant differences were evident between disease duration groups. ( $\mathbf{G}$ and $\mathbf{H}$ ) Quantification of CD34 staining stratified by disease duration demonstrates no relationship between microvascular density and disease duration. (I and J) Quantification of myocardial fibrosis (I) and cardiomyocyte cross-sectional area (J) stratified by disease duration reveals that children diagnosed with heart failure < 1 year or $<1$ year prior to tissue procurement displayed no indistinguishable levels of myocardial fibrosis and cardiomyocyte hypertrophy compared with donor controls. No significant differences were evident between disease duration groups. ${ }^{*} P<0.05$ compared with donor control. Mann Whitney $U$ test $(\mathbf{A}, \mathbf{C}, \mathbf{G})$ or $\chi^{2}$ test (E and $\left.\mathbf{F}\right)$.

to disease progression and need for cardiac transplantation or mechanical circulatory support (37). Interestingly, a competing-outcomes analysis demonstrated that, within 2 years of diagnosis, children with DCM will experience sustained disease stabilization, cardiac recovery, or disease progression (cardiac mortality or transplantation) (26). The mechanisms that orchestrate why individual children experience each of these outcomes remain poorly defined.

One of the most apparent distinctions between pediatric and adult DCM is the repeated observation that antiremodeling medications routinely used to treat adult heart failure patients do not significantly improve outcomes in children. The Pediatric Carvedilol Trial failed to demonstrate significant improvements in either clinical outcomes or echocardiographic parameters in children with DCM (7). In addition, registry data revealed that children with DCM treated with angiotensin converting enzyme (ACE) inhibitors or $\beta$-blockers had indistinguish-

able survival compared with those only receiving digoxin- and diuretic-based regimens $(1,9)$. Our data provides a mechanistic rationale for why children do not respond to adult heart failure therapeutics and strongly suggest that prognostic and therapeutics strategies routinely used in adults may have limited clinical utility in the pediatric population. These results are consistent with prior studies showing that hallmarks of adverse remodeling, including $\beta$-adrenergic receptor downregulation, fetal gene expression, and fibrosis, occur to a lesser extent in pediatric DCM $(10,38)$. In addition, the lack of myocardial fibrosis in pediatric patients with DCM may provide an explanation as to why sudden death is more prevalent in adult compared with pediatric DCM patients (39).

Disease duration represented a potential important variable that differed between the pediatric and adult DCM cases. Controlling for disease duration in our adult cohort demonstrated that increased disease duration in adult DCM did not constitute the primary reason why cardiomyocyte hypertrophy and myocar- 
Table 4. GO pathway analysis

\begin{tabular}{|c|c|c|c|}
\hline Increased expression in adult DCM, $n=1,018$ & genes & $P$ value & Fold enrichment \\
\hline GO:0055114: oxidation reduction & 67 & $2.40 \times 10^{-7}$ & 1.94 \\
\hline GO:0009719: response to endogenous stimulus & 47 & $1.35 \times 10^{-6}$ & 2.15 \\
\hline G0:0009611: response to wounding & 51 & $8.05 \times 10^{-5}$ & 1.78 \\
\hline GO:0006954: inflammatory response & 35 & $1.66 \times 10^{-4}$ & 2.00 \\
\hline G0:0006631: fatty acid metabolic process & 25 & $1.71 \times 10^{-4}$ & 2.33 \\
\hline CO:0007155: cell adhesion & 54 & $5.56 \times 10^{-8}$ & 2.23 \\
\hline G0:0055085: transmembrane transport & 43 & $2.77 \times 10^{-6}$ & 2.18 \\
\hline G0:0007601: visual perception & 19 & $5.06 \times 10^{-4}$ & 2.54 \\
\hline
\end{tabular}

dial fibrosis occurred at dramatically higher frequency in adult DCM patients. However, there was a trend $(P=0.09)$ between increased disease duration and greater extents of cardiomyocyte hypertrophy in adult DCM. Examination of disease duration in the pediatric DCM groups revealed no significant relationship between disease duration, cardiomyocyte hypertrophy, or adverse remodeling. Of note, statistically nonsignificant trends suggested that cardiomyocyte hypertrophy may be exaggerated by hypertension and chronic kidney disease. These data suggest that, within the adult DCM cohort, disease duration, hypertension, and chronic kidney disease may contribute to the extent of adverse remodeling. Importantly, these relationships were limited by sample size and should be viewed as speculative.

The apparent absence of adverse remodeling in pediatric DCM raises the question of what distinct disease mechanisms drive heart failure progression in children. In adult patients with ischemic and dilated cardiomyopathies, it is clear that heart failure initiation and progression are driven by separate pathophysiological processes (i.e., myocardial infarction or genetic mutations and adverse remodeling, respectively) $(40,41)$. Whether the failing pediatric heart follows a similar paradigm and undergoes a distinct remodeling program or whether disease progression is simply governed by the underlying cause of the cardiomyopathy is unclear. Future studies clarifying these mechanisms will be essential to guide effective therapeutic strategies for children with DCM.

The capacity to recover cardiac function (i.e., cardiac recovery), represents an important difference between pediatric and adult DCM. Approximately 20\% of children diagnosed with pediatric DCM will spontaneously and completely recover LV systolic function over a two-year follow-up period (37). In contrast, cardiac recovery is a relatively infrequent outcome in the adult patients with chronic heart failure (42, 43). The observation that cardiac recovery occurs spontaneously in the pediatric population was further demonstrated in the Pediatric Carvedilol Trial, where children in both the placebo and carvedilol groups displayed improved LV systolic function through the course of the study (7). These observations suggest that cardiac recovery represents an achievable outcome and a potential target for pediatric heart failure therapeutics. Consistent with this concept, previous reports have indicated that patients who experience cardiac recovery have improved quality of life and long-term survival (44).

The underlying mechanisms that dictate cardiac recovery and explain why children have a greater capacity for cardiac recovery compared with adults are poorly defined. The absence of adverse remodeling in pediatric DCM patients may represent one such explanation. Indeed, we and others have previously demonstrated that, in a mouse model of pediatric cardiac recovery, adverse remodeling only occurs in animals that lack the capacity to replace lost cardiomyocytes and expand the coronary vasculature (45-47). Consistent with these animal models, it has previously been demonstrated that children have a greater capacity for cardiomyocyte proliferation compared with adults (48), and in this study, we showed that coronary microvascular expansion occurs in pediatric but not adult DCM.

The molecular basis explaining why pediatric and adult DCM have differential capacity for cardiac recovery and distinct pathologies (i.e., adverse remodeling) are poorly defined. Using RNA sequencing, we compared the transcriptomes of 18 pediatric and 33 adult DCM patients, the largest study reported to date, to our knowledge. Strikingly, more than 1,900 mRNA transcripts were differentially regulated between pediatric and adult DCM using an FDR $<0.05$ and fold change $>1.5$. In addition to differential expression of transcripts associated with 
A
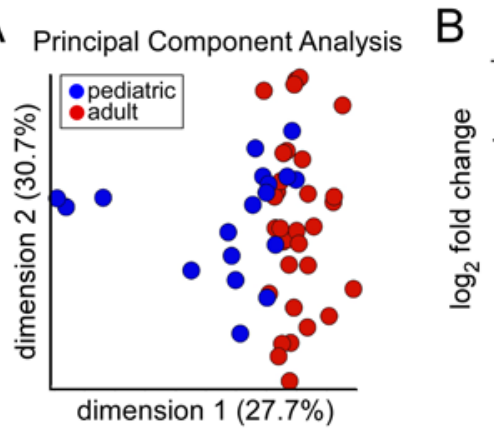

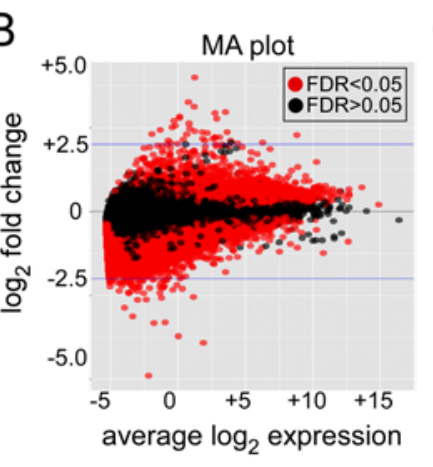

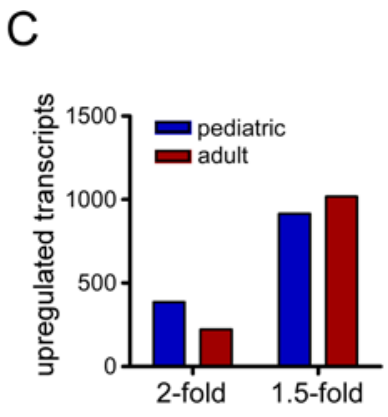

E

$\mathrm{D}$

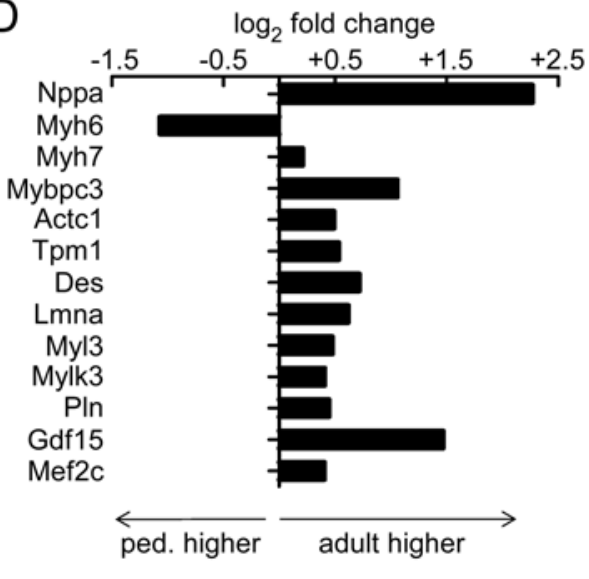

$\log _{2}$ fold change

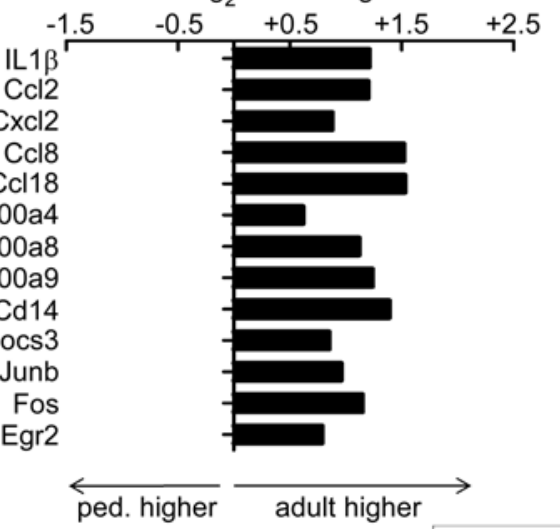

$\mathrm{F}$

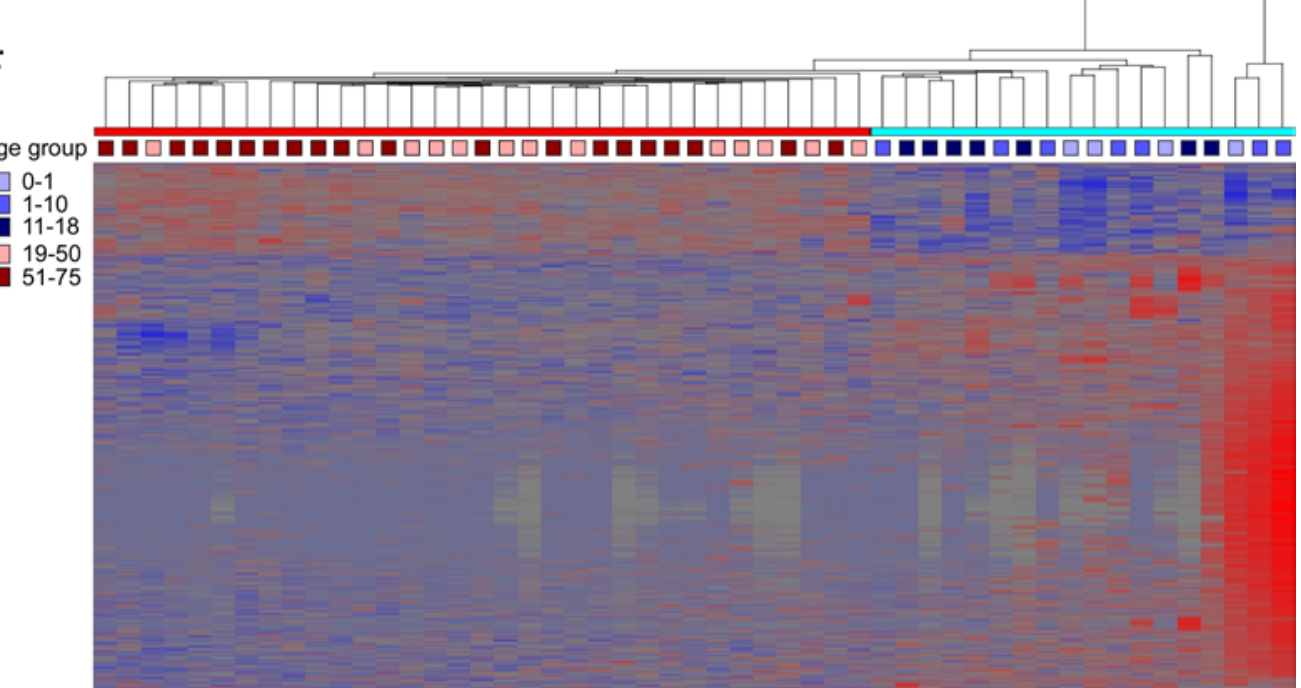

Figure 6. RNA sequencing analysis. (A) Principal component analysis demonstrating segregation of pediatric and adult DCM samples. (B) Bland-Altman (MA) plot depicting the relationship between differential gene expression and transcript abundance in pediatric and adult DCM samples. (C) Differential gene expression analysis highlighting the number of mRNA transcripts that are differentially expressed by 1.5- and 2-fold in pediatric and adult DCM samples. (D and $\mathbf{E})$ mRNA transcripts associated with sarcomere remodeling (D) and inflammation (E) are differentially regulated in pediatric and adult DCM. (F) Hierarchical clustering analysis illustrating that pediatric and adult DCM samples cluster as two distinct entities rather than as a continuum of age. Ped, pediatric.

adverse remodeling, pathway analysis revealed that adult specimens displayed marked induction of transcripts associated with innate immune system activation, fatty acid and oxidative metabolism, and metabolite signaling. These data confirm the importance of metabolism and substrate utilization in adult heart failure and signify the potential relevance of innate immune activation in the pathogenesis of adult DCM.

A wealth of literature has established that the failing adult heart displays important restrictions in substrate availability and utilization (49). Under resting conditions, the healthy adult heart metabolizes a diverse array of substrates including glucose, fatty acids, amino acids, and ketones. When the healthy heart 
is faced with periods of stress, glucose and, to a lesser extent, free fatty acid utilization increases (50). In contrast, metabolic derangements in the chronically failing adult heart result in restricted substrate utilization. While fatty acid oxidation is maintained in early stages of adult heart failure $(51,52)$, reductions in fatty acid uptake and utilization occur during the course of disease progression (53). Consistent with the loss of metabolic flexibility, the chronically failing adult heart is unable to augment glucose uptake during periods of stress $(54,55)$. These metabolic perturbations are thought to be due to a complex interplay of mechanisms, including insulin resistance, mitochondrial dysfunction, and neurohormonal activation (56, 57). Interestingly, increased sympathetic outflow and plasma norepinephrine levels in adult heart failure are associated with the development of insulin resistance, circulating fatty acids, and impaired glucose utilization linking metabolic derangements to adverse remodeling (58). With the exception of differing sensitivities to ischemia (33), little is known regarding metabolism in the pediatric heart.

Previous studies have established that innate immune activation is associated with the onset and progression of heart failure in adult patients. Circulating levels of TNF $\alpha, \operatorname{IL} 1 \beta$, and IL6 are elevated in adults with chronic heart failure and are predictive of poor prognosis (59-63). While the precise mechanisms that orchestrate proinflammatory responses in adult heart failure are not completely understood, it is clear that inhibition of inflammatory cytokine signaling may be beneficial (64). Blockade of IL1 $\beta$ signaling improves LV systolic function and suppresses components of adverse remodeling, including cardiomyocyte hypertrophy and myocardial fibrosis (65-70). As such, IL1 $\beta$ antagonists are under clinical investigation as novel therapeutics for adults with acute myocardial infarction (MI) and heart failure $(71,72)$. While reduced inflammatory gene expression in pediatric DCM may suggest that antiinflammatory approaches may be less efficacious in children, future studies will be required to directly test the feasibility of IL1 $\beta$ blockade in pediatric heart failure.

In comparison with adult DCM, pediatric specimens displayed induction of transcripts associated with cell adhesion, ion and transmembrane transport, and a variety of novel G-protein coupled receptors similar to those utilized for visual perception. Based on the current literature, the functional relevance of these genes and pathways with respect to the pathophysiology of pediatric heart failure is not immediately apparent. Whether these gene expression changes represent adaptive or maladaptive responses to pediatric DCM remains unclear but will undoubtedly be the subject of future investigation in suitable preclinical models.

Limitations. While this study represents, to our knowledge, the largest cohort of pediatric and adult DCM specimens studied to date, sample size remains an important limitation. Although the overall pediatric and adult populations were of sufficient size for comparative analyses, secondary subgroup analyses (i.e., age subgroups) were limited by sample size and, as such, should be interpreted with caution. In addition, this analysis is limited by the availability of clinical data, including genetic analyses identifying causative variants. Thus, covariates in addition to patient age may contribute to differences in adverse remodeling and gene expression. For example, in the absence of genetic data, it is not possible to directly compare pediatric and adult DCM samples harboring similar mutations. However, given the robust differences observed across pediatric and adult cohorts, it is likely patient age (pediatric vs. adult) represents a major component. The omission of control specimens represents an inherent limitation of our RNA sequencing analysis. As control specimens are typically obtained from brain-dead donors that are not suitable for transplantation due to preexisting cardiac disease, hepatitis C/HIV, prolonged ischemic times, and end organ failure, they do not represent normal cardiac tissue. While these tissues do not appear to demonstrate chronic pathological changes, they likely have significant derangements in gene expression, and that would likely confound our RNA sequencing analysis. For this reason, we excluded donor controls from the RNA sequencing analysis and recognize age as a confounding variable. Lastly, it is currently unknown whether our findings extend to other etiologies of pediatric heart failure, including hypertrophic cardiomyopathy, chemotherapy-induced cardiomyopathy, or even single-ventricle myocardial dysfunction. Future studies should investigate whether and to what extent adverse remodeling occurs in these disease settings.

Conclusions. Collectively, our findings support the concept that pediatric and adult DCM represent distinct pathological entities and provide a mechanistic basis to explain why children with DCM fail to respond to antiremodeling medications commonly used in adult heart failure patients. Together, these observations support a shift in clinical management of pediatric DCM and suggest that it may be appropriate to revise therapeutic approaches for children stricken with this devastating disease. 


\section{Methods}

Pathologic specimens. Cardiac tissue specimens were obtained from pediatric and adult patients with idiopathic and familial DCM undergoing LVAD implantation or cardiac transplantation. Patients with secondary causes of DCM, including cardiac amyloidosis, cardiac sarcoidosis, viral myocarditis, giant cell myocarditis, peripartum cardiomyopathy, and complex congenital heart disease, were excluded from this study. In addition, children who were classified as having neuromuscular disease-related cardiomyopathy, metabolic/mitochondrial disorder, or myocarditis on the basis of clinical presentation or biopsy were excluded. Adults with ischemic cardiomyopathy, chemotherapy-associated cardiomyopathy, and alcohol-induced cardiomyopathy were excluded. Tissues consisted of transmural specimens obtained from the apical or lateral wall of the LV. Cardiac tissue specimens (donor control and DCM) were either immediately flash frozen or fixed in 10\% formalin upon collection to preserve tissue integrity. Pediatric DCM specimens were acquired from the PCMR tissue repository and the Washington University Translational Cardiovascular Biobank and Repository (TCBR). Pediatric donor control specimens were obtained from the University of Colorado (Boulder, Colorado, USA). Control specimens acquired from brain-dead patients with normal LV systolic function and LV dimensions by echocardiography that were deemed not suitable for cardiac transplantation. Adult donor control and DCM specimens were obtained from the TCBR. The PCMR repository and the TCBR tissue bank diagnostic criteria for DCM was based on echocardiographic measurements related to LV enlargement and depressed systolic function, pathologic findings at autopsy, explanation or biopsy, and clinical evidence by the diagnosing cardiologist.

Cardiomyocyte hypertrophy. To evaluate for cardiomyocyte hypertrophy, rhodamine-conjugated WGA staining was used to demarcate plasma membrane boundaries on tissue sections of cardiomyocytes in cross section. Myocardial specimens were fixed in 10\% formalin for 72 hours. Tissues were embedded in paraffin and $4-\mu \mathrm{m}$ sections obtained using a Leica microtome. Paraffin sections were dewaxed with xylene, rehydrated, blocked with 1\% BSA, and stained rhodamine-conjugated WGA (Vector Laboratories, 1:200). Sections were then washed and mounted in Vectashield with DAPI (Vector Laboratories). At least 5 images per specimen were obtained on a Zeiss Confocal Microscope at $20 \times$ magnification. Serial sections were used to identify cardiomyocytes that were oriented perpendicular to the long axis. Cross-sectional area was measured using AxioVision software. For each individual, at least two separate myocardial specimens were examined and $>20$ cardiomyocytes per specimen were measured by two observers blinded to the experimental group. The criteria for cardiomyocytes to be included in the analysis consisted of cross-sectional morphology and the presence of DAPI-stained nuclear material.

Myocardial fibrosis. To evaluate for myocardial fibrosis, we stained for collagen using Picrosirius red (Thermo Fisher Scientific). Paraffin-embedded sections were dewaxed in xylene, rehydrated, stained with Picrosirius red for 20 minutes, washed in acid water, dehydrated, and mounted in a xylene-based media (Cytoseal, Thermo Fisher Scientific). For each individual, multiple fields from at least two separate myocardial specimens were included in the final analysis. A scoring system was utilized to measure the degrees of perivascular fibrosis and interstitial fibrosis to allow discrimination of distinct patterns of fibrosis. Scoring was performed by two observers blinded to the experimental group, with results averaged for each patient. The scoring protocol is outlined in Supplemental Figure 1.

Capillary staining. To measure coronary microvascular density, we performed IHC with an anti-CD34 (QBEnd/10, Abcam) antibody (catalog ab8536). The CD34 antibody was chosen due to previous reports demonstrating excellent signal intensity across a variety of specimens (23). Paraffin-embedded sections were dewaxed in xylene, rehydrated, blocked in 1\% BSA, and stained with anti-CD34 (1:400) antibody overnight. The primary antibody was detected using a biotin conjugated anti-mouse secondary antibody (Vector Laboratories) in conjunction with streptavidin HRP (ABC Elite, Vector Laboratories) and DAB reagent (Vector Laboratories). Slides were then dehydrated and mounted in a xylene-based media (Cytoseal). Photographs were acquired at $20 \times$ on a Zeiss Axioskope system. For each individual, at least two separate myocardial specimens were included. Microvascular density was measured from at least 2-3 slides per specimen using Image J software as previously described (23). Using this methodology, we previously demonstrated that automated measurement of the percent area stained was highly correlated with manual counting of the number of capillaries per field. The averaged values from $>5$ images per individual were included in the final analysis.

Electron microscopy. Cardiac specimens $\left(2 \mathrm{~mm}^{2}\right)$ were fixed in a mixture of $2.5 \%$ glutaraldehyde and $2 \%$ paraformaldehyde in $0.15 \mathrm{M}$ cacodylate buffer at $\mathrm{pH} 7.4$ containing $2 \mathrm{mM}$ calcium chloride at $4^{\circ} \mathrm{C}$ overnight. Sample were rinsed in $0.15 \mathrm{M}$ cacodylate buffer $3 \times$ for 10 minutes each and subjected to a 
secondary fixation step for 1 hour in $1 \%$ osmium tetroxide containing $1.5 \%$ potassium ferrocyanide in cacodylate buffer on ice. Following fixation, samples were then washed in ultrapure water $3 \times$ for 10 minutes each and en bloc stained for 1 hour with $2 \%$ aqueous uranyl acetate. All reagents listed in previous sentences are from Sigma-Aldrich. After staining was complete, samples were briefly washed in ultrapure water, dehydrated in a graded acetone series $(50 \%, 70 \%, 90 \%, 100 \% \times 2)$ for 10 minutes in each step, infiltrated with microwave assistance (Pelco BioWave Pro) into LX112 resin, embedded in silicone molds, and cured in an oven at $60^{\circ} \mathrm{C}$ for 48 hours. Once the resin was cured, each block was trimmed and faced using a diamond trim tool (Leica EM TRIM2). Longitudinal sections (70 $\mathrm{nm}$ ) were obtained and imaged on a FE-SEM (Zeiss Crossbeam 540) using the aSTEM detector. The SEM was operated at $28 \mathrm{KeV}$ with a probe current of $0.9 \mathrm{nA}$, and the STEM detector was operated with the annular rings inverted for additional sample contrast. Large sample areas were imaged at a resolution of 4,096 $\times 3,072$ pixels, with a pixel size of $5.582 \mathrm{~nm}$. Sarcomere thickness was measured in two samples per specimen by individuals blinded to sample identity. Greater than 20 sarcomeres were measured from each individual patient sample, and the average sarcomere thickness was calculated.

RNA sequencing. Total RNA was harvested from transmural pediatric and adult DCM samples (200-400 mg) by disrupting samples in Trizol (Thermo Fisher Scientific) using the Qiagen TissueLyser homogenizer with stainless steel beads. Following phenol chloroform extraction, RNA was purified using the Ambion Purelink miniprep kit. RNA integrity was quantified on an Agilent Bioanalyzer, and samples with RNA Integrity Number $>8$ were utilized for RNA sequencing. Ribosomal RNAs were depleted with Ribo-Zero, cDNA libraries were generated, and samples were sequenced $(1 \times 50$ bp reads) on an Illumina HiSeq 3000 instrument. Sequence alignment, normalization, and differential expression analysis was carried out in the Genome Technology Access Center at Washington University using Limma-Voom software. PCA and Hierarchical cluster analysis was performed in Partek. GO Pathway analysis was performed using DAVID. Transcripts with $>10$ reads in $50 \%$ of samples demonstrating a fold change $>1.5$-fold at an FDR $<0.05$ were included in the pathway analysis. Raw data was deposited in the NCBI sequence reads archive (SRA, SRP108128).

Statistics. Primary comparisons were made between control and DCM groups for each age group. Fisher's exact and Mann-Whitney $U$ tests with appropriate Bonferroni correction were used to identify statistically significant differences between groups. Data are presented as either dot plots or bar graphs, with all data points displayed (mean $\pm \mathrm{SD})$.

Study approval. This study was approved by the Washington University in St. Louis IRB (protocal number 201305086). All subjects provided informed consent prior to sample collection, and the experiments were performed in accordance with the approved study protocol.

\section{Author contributions}

MDP performed and analyzed the quantitative histology and immunostaining experiments. JM performed and analyzed the RNA sequencing studies. CS and GB assisted with the immunostaining experiments. EP and JT provided pediatric LV myocardium specimens through the PCM biorepository and assisted with study design. $\mathrm{CEC}$ and $\mathrm{AB}$ assisted with study design, data interpretation, and manuscript production. $\mathrm{KJL}$ was responsible for all aspects of this study, including study design, experimental execution, data analysis, data interpretation, and manuscript production.

\section{Acknowledgments}

We acknowledge Brian L. Stauffer, Carmen C. Sucharov, Shelley D. Miyamoto, and the Pediatric Cardiovascular Research Laboratory for the pediatric donor samples. The authors are also grateful for support in sample preparation and Electron Microscopy provided by Matthew Joens and James Fitzpatrick. This project was made possible by funding provided from the Children's Discovery Institute of Washington University and St. Louis Children's Hospital (CH-II-2015-462, CH-II-2017-628), Foundation of Barnes-Jewish Hospital (8038-88), and Longer Life Foundation (2016-004). KL is supported by NIH K08 HL123519, R01 HL138466, and Burroughs Welcome Fund (1014782). Histology was performed in the DDRCC advanced imaging and tissue analysis core supported by grant P30 DK52574. The Genome Technology Access Center in the Department of Genetics at Washington University School of Medicine is partially supported by NCI Cancer Center Support grant P30 CA91842 to the Siteman Cancer Center and by ICTS/CTSA grant UL1TR000448 from the National Center for Research Resources (NCRR), a component of the NIH, and 
NIH Roadmap for Medical Research. The Washington University Center for Cellular Imaging is supported by Washington University School of Medicine, the Children's Discovery Institute of Washington University and St. Louis Children's Hospital (CDI-CORE-2015-505), the Foundation for Barnes-Jewish Hospital (3770) and the National Institute for Neurological Disorders and Stroke (NS086741).

Address for correspondence: Kory J. Lavine, Division of Cardiology, 660 South Euclid, Campus Box 8086, St. Louis, Missouri 63110, USA. Phone: 314.362.1171; Email: klavine@wustl.edu.

1. Towbin JA, et al. Incidence, causes, and outcomes of dilated cardiomyopathy in children. JAMA. 2006;296(15):1867-1876.

2. Taylor DO, et al. Registry of the International Society for Heart and Lung Transplantation: Twenty-sixth Official Adult Heart Transplant Report-2009. J Heart Lung Transplant. 2009;28(10):1007-1022.

3. Go AS, et al. Heart disease and stroke statistics--2013 update: a report from the American Heart Association. Circulation. 2013;127(1):e6-e245.

4. Rampersaud E, Siegfried JD, Norton N, Li D, Martin E, Hershberger RE. Rare variant mutations identified in pediatric patients with dilated cardiomyopathy. Prog Pediatr Cardiol. 2011;31(1):39-47.

5. Goldberg LR. In the clinic. Heart failure. Ann Intern Med. 2010;152(11):ITC61-15; quiz ITC616.

6. Burchfield JS, Xie M, Hill JA. Pathological ventricular remodeling: mechanisms: part 1 of 2. Circulation. 2013;128(4):388-400.

7. Shaddy RE, et al. Carvedilol for children and adolescents with heart failure: a randomized controlled trial. JAMA. 2007;298(10):1171-1179.

8. Bristow MR, et al. Carvedilol produces dose-related improvements in left ventricular function and survival in subjects with chronic heart failure. MOCHA Investigators. Circulation. 1996;94(11):2807-2816.

9. Kantor PF, Abraham JR, Dipchand AI, Benson LN, Redington AN. The impact of changing medical therapy on transplantation-free survival in pediatric dilated cardiomyopathy. J Am Coll Cardiol. 2010;55(13):1377-1384.

10. Miyamoto SD, et al. Beta-adrenergic adaptation in paediatric idiopathic dilated cardiomyopathy. Eur Heart J. $2014 ; 35(1): 33-41$.

11. Gerdes AM, et al. Structural remodeling of cardiac myocytes in patients with ischemic cardiomyopathy. Circulation. 1992;86(2):426-430.

12. Russell B, Curtis MW, Koshman YE, Samarel AM. Mechanical stress-induced sarcomere assembly for cardiac muscle growth in length and width. J Mol Cell Cardiol. 2010;48(5):817-823.

13. Lattouf R, et al. Picrosirius red staining: a useful tool to appraise collagen networks in normal and pathological tissues. $J$ Histochem Cytochem. 2014;62(10):751-758.

14. Klotz S, et al. Mechanical unloading during left ventricular assist device support increases left ventricular collagen cross-linking and myocardial stiffness. Circulation. 2005;112(3):364-374.

15. Rakusan K, Flanagan MF, Geva T, Southern J, Van Praagh R. Morphometry of human coronary capillaries during normal growth and the effect of age in left ventricular pressure-overload hypertrophy. Circulation. 1992;86(1):38-46.

16. Abraham D, et al. Selective downregulation of VEGF-A(165), VEGF-R(1), and decreased capillary density in patients with dilative but not ischemic cardiomyopathy. Circ Res. 2000;87(8):644-647.

17. Dashkevich A, et al. Immunohistochemical study of remodeling of myocardial lymphatic and blood microvascular structures in terminal heart failure: differences between ischemic and dilated cardiomyopathy. Lymphology. 2010;43(3):110-117.

18. Tsagalou EP, et al. Depressed coronary flow reserve is associated with decreased myocardial capillary density in patients with heart failure due to idiopathic dilated cardiomyopathy. J Am Coll Cardiol. 2008;52(17):1391-1398.

19. Djordjevic Dikic A, et al. Prognostic role of coronary flow reserve for left ventricular functional improvement after cardiac resynchronization therapy in patients with dilated cardiomyopathy. Eur Heart J Cardiovasc Imaging. 2014;15(12):1344-1349.

20. Sugioka K, et al. Relation of early improvement in coronary flow reserve to late recovery of left ventricular function after beta-blocker therapy in patients with idiopathic dilated cardiomyopathy. Am Heart J. 2007;153(6):1080.e1-1080.e6.

21. Rigo F, et al. The prognostic impact of coronary flow-reserve assessed by Doppler echocardiography in non-ischaemic dilated cardiomyopathy. Eur Heart J. 2006;27(11):1319-1323.

22. Rigo F, Gherardi S, Galderisi M, Sicari R, Picano E. The independent prognostic value of contractile and coronary flow reserve determined by dipyridamole stress echocardiography in patients with idiopathic dilated cardiomyopathy. Am J Cardiol. 2007;99(8):1154-1158.

23. Lavine KJ, et al. Coronary collaterals predict improved survival and allograft function in patients with coronary allograft vasculopathy. Circ Heart Fail. 2013;6(4):773-784.

24. Molina KM, et al. Predictors of disease progression in pediatric dilated cardiomyopathy. Circ Heart Fail. 2013;6(6):1214-1222.

25. Aleksova A, et al. Natural history of dilated cardiomyopathy: from asymptomatic left ventricular dysfunction to heart failure--a subgroup analysis from the Trieste Cardiomyopathy Registry. J Cardiovasc Med (Hagerstown). 2009;10(9):699-705.

26. Alvarez JA, et al. Competing risks for death and cardiac transplantation in children with dilated cardiomyopathy: results from the pediatric cardiomyopathy registry. Circulation. 2011;124(7):814-823.

27. Watts RP, Thom O, Fraser JF. Inflammatory signalling associated with brain dead organ donation: from brain injury to brain stem death and posttransplant ischaemia reperfusion injury. J Transplant. 2013;2013:521369.

28. Wilhelm MJ, et al. Activation of the heart by donor brain death accelerates acute rejection after transplantation. Circulation. 2000;102(19):2426-2433.

29. Haque ZK, Wang DZ. How cardiomyocytes sense pathophysiological stresses for cardiac remodeling. Cell Mol Life Sci. 2017;74(6):983-1000.

30. Schwientek P, et al. Global gene expression analysis in nonfailing and failing myocardium pre- and postpulsatile and nonpulsatile ventricular assist device support. Physiol Genomics. 2010;42(3):397-405. 
31. Hartupee J, Mann DL. Positioning of inflammatory biomarkers in the heart failure landscape. J Cardiovasc Transl Res. 2013;6(4):485-492.

32. Bozkurt B, Mann DL, Deswal A. Biomarkers of inflammation in heart failure. Heart Fail Rev. 2010;15(4):331-341.

33. Wittnich C, Belanger MP, Bandali KS. Newborn hearts are at greater 'metabolic risk' during global ischemia--advantages of continuous coronary washout. Can J Cardiol. 2007;23(3):195-200.

34. Lai L, et al. Energy metabolic reprogramming in the hypertrophied and early stage failing heart: a multisystems approach. Circ Heart Fail. 2014;7(6):1022-1031.

35. Wilkinson JD, et al. The pediatric cardiomyopathy registry and heart failure: key results from the first 15 years. Heart Fail Clin. 2010;6(4):401-13, vii.

36. Wilkinson JD, Westphal JA, Bansal N, Czachor JD, Razoky H, Lipshultz SE. Lessons learned from the Pediatric Cardiomyopathy Registry (PCMR) Study Group. Cardiol Young. 2015;25 Suppl 2:140-153.

37. Everitt MD, et al. Recovery of echocardiographic function in children with idiopathic dilated cardiomyopathy: results from the pediatric cardiomyopathy registry. J Am Coll Cardiol. 2014;63(14):1405-1413.

38. Woulfe KC, et al. Fibrosis and Fibrotic Gene Expression in Pediatric and Adult Patients With Idiopathic Dilated Cardiomyopathy. J Card Fail. 2017;23(4):314-324.

39. Pahl E, et al. Incidence of and risk factors for sudden cardiac death in children with dilated cardiomyopathy: a report from the Pediatric Cardiomyopathy Registry. J Am Coll Cardiol. 2012;59(6):607-615.

40. Hartupee J, Mann DL. Neurohormonal activation in heart failure with reduced ejection fraction. Nat Rev Cardiol. 2017;14(1):30-38.

41. Mann DL. Basic mechanisms of left ventricular remodeling: the contribution of wall stress. J Card Fail. 2004;10(6 Suppl):S202-S206.

42. Mann DL, Barger PM, Burkhoff D. Myocardial recovery and the failing heart: myth, magic, or molecular target? J Am Coll Cardiol. 2012;60(24):2465-2472.

43. Mann DL, Bogaev R, Buckberg GD. Cardiac remodelling and myocardial recovery: lost in translation? Eur J Heart Fail. 2010;12(8):789-796.

44. McNamara DM, et al. Clinical and demographic predictors of outcomes in recent onset dilated cardiomyopathy: results of the IMAC (Intervention in Myocarditis and Acute Cardiomyopathy)-2 study. J Am Coll Cardiol. 2011;58(11):1112-1118.

45. Porrello ER, et al. Transient regenerative potential of the neonatal mouse heart. Science. 2011;331(6020):1078-1080

46. Aurora AB, et al. Macrophages are required for neonatal heart regeneration. J Clin Invest. 2014;124(3):1382-1392.

47. Lavine KJ, et al. Distinct macrophage lineages contribute to disparate patterns of cardiac recovery and remodeling in the neonatal and adult heart. Proc Natl Acad Sci U S A. 2014;111(45):16029-16034.

48. Mollova M, et al. Cardiomyocyte proliferation contributes to heart growth in young humans. Proc Natl Acad Sci U S A. 2013;110(4):1446-1451.

49. Wang ZV, Li DL, Hill JA. Heart failure and loss of metabolic control. J Cardiovasc Pharmacol. 2014;63(4):302-313.

50. Taegtmeyer H, Golfman L, Sharma S, Razeghi P, van Arsdall M. Linking gene expression to function: metabolic flexibility in the normal and diseased heart. Ann N Y Acad Sci. 2004;1015:202-213.

51. Funada J, et al. Substrate utilization by the failing human heart by direct quantification using arterio-venous blood sampling. PLoS One. 2009;4(10):e7533.

52. Paolisso G, et al. Total-body and myocardial substrate oxidation in congestive heart failure. Metab Clin Exp. 1994;43(2):174-179.

53. Yazaki Y, et al. Assessment of myocardial fatty acid metabolic abnormalities in patients with idiopathic dilated cardiomyopathy using 123I BMIPP SPECT: correlation with clinicopathological findings and clinical course. Heart. 1999;81(2):153-159.

54. Neglia D, et al. Impaired myocardial metabolic reserve and substrate selection flexibility during stress in patients with idiopathic dilated cardiomyopathy. Am J Physiol Heart Circ Physiol. 2007;293(6):H3270-H3278.

55. Lionetti V, et al. Mismatch between uniform increase in cardiac glucose uptake and regional contractile dysfunction in pacing-induced heart failure. Am J Physiol Heart Circ Physiol. 2007;293(5):H2747-H2756.

56. Abel ED, Doenst T. Mitochondrial adaptations to physiological vs. pathological cardiac hypertrophy. Cardiovasc Res. 2011;90(2):234-242

57. Lionetti V, Stanley WC, Recchia FA. Modulating fatty acid oxidation in heart failure. Cardiovasc Res. 2011;90(2):202-209.

58. Triposkiadis F, Karayannis G, Giamouzis G, Skoularigis J, Louridas G, Butler J. The sympathetic nervous system in heart failure physiology, pathophysiology, and clinical implications. J Am Coll Cardiol. 2009;54(19):1747-1762.

59. Kapadia SR, Yakoob K, Nader S, Thomas JD, Mann DL, Griffin BP. Elevated circulating levels of serum tumor necrosis factor-alpha in patients with hemodynamically significant pressure and volume overload. J Am Coll Cardiol. 2000;36(1):208-212.

60. Mann DL. Innate immunity and the failing heart: the cytokine hypothesis revisited. Circ Res. 2015;116(7):1254-1268.

61. Rauchhaus M, et al. Plasma cytokine parameters and mortality in patients with chronic heart failure. Circulation. 2000;102(25):3060-3067.

62. Torre-Amione G, Kapadia S, Benedict C, Oral H, Young JB, Mann DL. Proinflammatory cytokine levels in patients with depressed left ventricular ejection fraction: a report from the Studies of Left Ventricular Dysfunction (SOLVD). J Am Coll Cardiol. 1996;27(5):1201-1206.

63. Tsutamoto T, et al. Interleukin-6 spillover in the peripheral circulation increases with the severity of heart failure, and the high plasma level of interleukin-6 is an important prognostic predictor in patients with congestive heart failure. J Am Coll Cardiol. 1998;31(2):391-398.

64. Topkara VK, et al. Therapeutic targeting of innate immunity in the failing heart. J Mol Cell Cardiol. 2011;51(4):594-599.

65. Mezzaroma E, et al. The inflammasome promotes adverse cardiac remodeling following acute myocardial infarction in the mouse. Proc Natl Acad Sci U S A. 2011;108(49):19725-19730.

66. Abbate A, et al. Interleukin-1beta modulation using a genetically engineered antibody prevents adverse cardiac remodelling following acute myocardial infarction in the mouse. Eur J Heart Fail. 2010;12(4):319-322.

67. Abbate A, et al. Alterations in the interleukin-1/interleukin-1 receptor antagonist balance modulate cardiac remodeling following myocardial infarction in the mouse. PLoS One. 2011;6(11):e27923.

68. Toldo S, et al. Interleukin-1 $\beta$ blockade improves cardiac remodelling after myocardial infarction without interrupting the inflam- 
masome in the mouse. Exp Physiol. 2013;98(3):734-745.

69. Toldo S, et al. Interleukin-1 $\beta$ blockade improves left ventricular systolic/diastolic function and restores contractility reserve in severe ischemic cardiomyopathy in the mouse. J Cardiovasc Pharmacol. 2014;64(1):1-6.

70. Van Tassell BW, et al. Interleukin-1 trap attenuates cardiac remodeling after experimental acute myocardial infarction in mice. $J$ Cardiovasc Pharmacol. 2010;55(2):117-122.

71. Abbate A, et al. Interleukin-1 blockade with anakinra to prevent adverse cardiac remodeling after acute myocardial infarction (Virginia Commonwealth University Anakinra Remodeling Trial [VCU-ART] Pilot study). Am J Cardiol. 2010;105(10):1371-1377.e1.

72. Van Tassell BW, et al. Effects of interleukin-1 blockade with anakinra on aerobic exercise capacity in patients with heart failure and preserved ejection fraction (from the D-HART pilot study). Am J Cardiol. 2014;113(2):321-327. 